Article

\title{
Sensory-processing sensitivity and pathways to depression and aggression: the mediating role of trait emotional intelligence and decision-making style. A pilot study.
}

\author{
Nikola Drndarević ${ }^{1 *}$, Sonja Protić ${ }^{2}$ and José M. Mestre ${ }^{3, *}$ \\ 1 Institute of criminological and sociological research, Belgrade, Serbia; nikola.drndarevic@iksi.ac.rs \\ 2 Institute of criminological and sociological research, Belgrade, Serbia; and International Psychoanalytic \\ University, Berlin; sonja.milojevic@iksi.ac.rs; sonja.protic@ipu-berlin.de \\ 3 University Institute of Social and Sustainable Development (INDESS), Department of Psychology, Univer- \\ sity of Cádiz; josemi.mestre@uca.es \\ * Correspondence: nikola.drndarevic@iksi.ac.rs; josemi.mestre@uca.es
}

\begin{abstract}
While the link between Sensory-processing sensitivity (SPS) and internalizing symptoms has been well-established, a link to externalizing problems is still to be explored. This study aimed to further examine the relation between SPS and behavioral problems by testing the potential mediating roles of trait emotional intelligence (TEI) and decision-making styles. Pathway analyses were conducted on data from 268 community sample participants $\left(M_{\text {age }}=25.81, S D=2.41,61.2 \%\right.$ females). Results indicated gender differences in the pathway level outcomes of SPS, as well as potential partial mediators in men and women. SPS both directly and via the mediating effects of wellbeing factor (TEI) and avoidant decision-making influenced depression, regardless of gender. Direct effects on aggression were, however, obtained only in the male sample. Indirect effects of SPS on aggression were found in spontaneous decision-making for men, and in self-control and sociability factors of TEI for women. Directions for future research were discussed.
\end{abstract}

Keywords: sensory-processing sensitivity, depression, aggression, emotional intelligence, decisionmaking style, gender differences

\section{Introduction}

Sensory-processing sensitivity (SPS) represents a personality trait characterized by a greater depth of cognitive processing and greater emotional reactivity [1,2]. SPS involves increased, biologically-based, sensitivity to environmental stimuli $[1,2]$. Recently, there has been an increased theoretical and practical interest and recognition of the importance of SPS as an essential factor participating in both well-being and difficulties among more sensitive individuals, having implications on health, education and work [2]. Indeed, SPS could act as an advantage or a disadvantage, depending on whether the environments are supportive or adverse [2,3]. While supportive environments may help more sensitive individuals prosper, adverse environments seem to generate increased negative mental health outcomes, which may be manifested in problem behaviors [3].

Existing research has mainly focused on the relation between SPS and internalizing (INT) problem behavior [4], specifically with depression, anxiety, autism, alexithymia, and somatic problems [5-8]. The main representative of INT problems, depression [9], has revealed low-moderate positive associations (from 0.22 to 0.35 ) with more sensitive people $[5,6,10]$. Neal et al. [7] study did not find correlation between SPS and depression, which may correspond to SPS not being solely a disadvantage. 
Established findings of SPS and problem behaviors on INT spectrum were not surprising and indeed SPS has been characterized by the general avoidance strategy [1-3]. However, there are studies showing that greater susceptibility to environmental stimuli may result in both INT and externalizing (EXT) reaction [11]. Should SPS be associated with EXT reactions, this would raise the doubt to general avoidance strategy and pauseto-check behaviors assumed present in those higher in sensitivity [2,3]. Thus, the relationship between SPS and EXT problems remains insufficiently explored. To our knowledge, one study experimentally examined the role of SPS and EXT behaviors in a sample of children, which reported that highly sensitive children showed more EXT behavior problems [11]. No studies have explored yet the association between SPS and EXT problems, and specifically aggression, in older samples.

Notwithstanding the relevance of adverse environments, the mechanisms underlying SPS and psychological distress have been mostly neglected in literature [2]. More recently, a psychological model of cognitive reactivity has been suggested as a theoretical explanation for depression, anxiety and somatic problems in more sensitive individuals [12]. Viewed from this model, depression should not be seen as a consequence of stimuli or negative emotions, but rather as a secondary phenomenon of cognitive reactivity. In other words, SPS amplifies the negative aspects of situations, stimuli, and cognition increasing the probability of depression symptoms. However, it is not clear how this model would explain the potential relation of SPS to EXT outcomes. Moreover, considering the core facets of SPS (cognitive and emotional processing), it becomes relevant to explore factors belonging to these domains which may further our understanding of problem behaviors in more sensitive individuals. Thus, it was conceivable that the emotional factor of trait emotional intelligence and cognitive factor of decision-making may function as mediators between SPS and problem behaviors.

\subsection{Trait emotional intelligence as a mediator between SPS and problem behaviors}

The trait emotional intelligence (TEI) represents a pattern of behavioral dispositions and self-perceptions concerning individual's ability to recognize, process, and utilize emotion-laden information [13]. TEI consists of four global factors [14]: well-being (optimism, happiness and self-esteem), self-control (impulse control, emotional regulation and stress management), emotionality (emotional perception and expression and relationship skills), and sociability (social competence, assertiveness and affecting others' emotion). The current conceptualization of TEI refers to emotional self-efficacy skills that can be subjected to learning and experience, which can serve as a protective factor against various health and behavioral problems [13].

Surprisingly, to our knowledge, TEI construct has not been used in SPS research considering its range of covering one of the core facets of SPS as well as being implicated in various behavioral problems. Indirect research may be used as a guide to such relationship: SPS was related to lower well-being [15]; poor stress management and difficulties in emotion regulation [16]; as well as social and communication deficits [6]. The relationship between emotionality and SPS is ambiguous, considering the mentioned difficulties in emotion regulation, social deficits and lower self-esteem [3] on the one hand, and higher supposed emotion perception in SPS on the other [1]. Thus, SPS was expected to affect the TEI factors, especially well-being, self-control and sociability.

In addition, previous research [17] has indicated a link between TEI and INT (f.i., with depression [18]) and EXT (f.i., aggression [19]) problems. Individual TEI factors were rarely researched, except for one study which found negative correlations of each TEI factor and depression [18]. Few studies point to mixed-results when considering social deviance. Both high and low emotionality, low self-control and high sociability has been 
connected with social deviance [20,21]. Nevertheless, the research remains scarce [21], indicating the need for exploration within the mentioned domains.

In sum, the proposed model assumed that SPS directly influences problem behaviors and at least partially through well-being, self-control and sociability factors of TEI.

\subsection{Decision-making style as a mediator between SPS and problem behavior}

Decision-making styles are conceptualized as learned response patterns of behavior that can be used across contexts and decision situations, and are indicative of cognitive style of an individual [22]. Authors delineated five decision-making styles: avoidant (avoiding decision-making due to lack of confidence); spontaneous (characterized by a sense of immediacy and impulsiveness); intuitive (iteratively developed reliance on hunches and feelings); rational (systematic and logical approach to situations) and dependent (searching for advice and direction from others).

It has been suggested that SPS might affect decision-making, resulting in enhanced or diminished decision-making [3]. Furthermore, SPS individuals have been characterized as adopting a general avoidance strategy [2,3]. On the other hand, there is a lack of studies concerning decision-making and SPS, whereas its participation in developing problem behavior or psychopathology is well documented [23]. Although intuitive decision-making was expected in those higher in SPS [1], it was not clear how this would participate in problem behaviors, and together with rational and dependent decision-making were not the main focus of the study.

Concerning decision-making and problem behaviors, impaired decision-making may function as a factor amplifying the effects toward INT or EXT problems [24]. Previous research has indicated a link between avoidant decision-making and depression [25]. Due to greater sensitivity to risk, the avoidant decision-making style was present in depressive individuals [26]. Conversely, increased risk-taking and impulsive decision-making have been connected with higher levels of aggression [27]. Impulsivity has been connected to decision-making deficits and proneness to aggressive behavior [28], while EXT behaviors were associated with disinhibited decision-making [29].

In sum, the proposed model assumed that SPS directly influences problem behaviors and, at least partially: depression through avoidant decision-making and aggression through spontaneous (impulsive) decision-making styles.

\subsection{Role of gender as a moderating factor}

Previous research has consistently shown that women score higher on SPS than men [30]. It has been suggested that reported greater sensitivity in women may be due to differences in upbringing and socialization [1]. In comparison to women who report more INT, men report more EXT behavior [31], i.e. greater aggression in men and depression in women was frequently found [9].

Regarding gender and TEI, few studies have examined this relationship with no consensus [17]. A relatively consistent finding was reflected in women scoring higher in emotionality [18] and men scoring higher in self-control and sociability [17]. No difference was registered in the well-being factor [32]. The emotionality factor may lead to a higher prevalence of depressive symptoms (INT) in women [33]. Moreover, EXT outcomes and TEI are moderated with respect to sample, age, and gender. For example, in a sample of male juvenile offenders, lower well-being, self-control and emotionality was registered with social deviance [20]. In the community sample, both high and low emotionality, low self- 
control and high sociability have been connected with social deviance [21]. Additionally, social deviance TEI profiles point to inconsistency of TEI in the transitional period of adolescence toward adulthood [21], posing further restrictions on prediction.

The literature on decision-making and gender is not in unison. On the one hand, several research studies showed no gender difference in decision-making [22]. On the other hand, women seem to be more averse to risky choices and showed a stronger tendency to adopt an avoidant decision-making style than men [34]. It has been suggested that the cultural pressures shape decision-making in men and women, where taking risky behavior is unfavored for women, while the opposite is encouraged for men [35].

\subsection{Rationale}

Previous findings have linked SPS to problem behaviors, predominantly INT behavior [4]. However, to our knowledge, only one study examined environmental sensitivity and EXT behavior [11]. In light of this, we aimed to expand the research of SPS and EXT behavior, specifically aggression. Due to their connections with problem behaviors, TEI and decision-making could act as the potential candidates for understanding the relationship between SPS and problem behaviors. Consequently, the present study sought to explore the mediating roles of TEI (emotive factor) and decision-making styles (cognitive factor), in the relationship of SPS and depression and aggression.

Based on previous research, we construed a model where: (1) SPS is associated with and is directly influencing problem behaviors, both depression [5-7] and aggression [11], (2) SPS is indirectly influencing problem behaviors, reflected in partial mediating roles of TEI [16] and decision-making styles [3]. More specifically, we hypothesized that well-being factor of TEI partially mediates SPS and depression [3,4,16], while self-control and sociability partially mediate SPS and aggression [20]. As previously argued, the findings in literature concerning emotionality and well-being in relation to aggression are ambiguous, warranting further exploration. Moreover, regarding decision-making styles, factor differentiating a pathway toward depression is reflected in avoidant and a pathway toward aggression in spontaneous decision-making. Finally, we were interested in exploring the potential moderating influences of gender for the proposed pathways.

\section{Materials and Methods}

\subsection{Participants}

The study included a total of 268 participants that fully completed the questionnaire packet $(61.2 \%(\mathrm{n}=164)$ were women). The respondents age varied from 17 to 30 years with the mean age of 25.81 years (Median $=26$, Mode $=26, S D=2.41$ ). Education was represented by three levels: 1 . Middle or Higher school (18.3\%); 2. Bachelor studies (44\%); 3. Master studies (37.7\%);

\subsection{Procedure}

The study was approved by the Ethics Commission of the Faculty of Philosophy, University of Belgrade and was conducted with the help of an online platform, using convenience and snowball sampling techniques. The time estimated for questionnaire completion was between 15 and 25 minutes. In order to ensure more significant variability in scores, respondents from social networks and forums for people with higher sensitivity were also recruited. Respondents participated voluntarily, and the standards of informing, confidentiality, anonymity, and data retention were met. 


\subsection{Instruments}

The Highly Sensitive Person Scale (HSPS [1]). HSPS consisted of a 27-item scale in which participants were asked to rate their agreement with various statements on a Likert Scale ranging from 1 (strongly disagree) to 7 (strongly agree). Items reflected sensitivity to both internal and external stimuli (e.g., "Are you made uncomfortable by loud noises?"). Recent research suggests SPS being a continuous trait (Greven et al. 2019). Higher scores indicated greater sensory-processing sensitivity. The test items were adapted from English to Serbian using the double-translation procedure.

General Decision-Making Style (GDMS [22]). GDMS consisted of 25 items in which participants were asked to rate the items on a five-point Likert-type scale (e.g., "I make quick decisions."). The items measured five decision-making styles (rational, dependent, intuitive, avoidant, spontaneous), each consisting of five items. The test items were adapted from English to Serbian using the double-translation procedure.

Trait Emotional Intelligence Questionnaire-Short Form (TEIQue-SF [14]; the Serbian version of the Trait Emotional Intelligence Questionnaire [36]). TEI was assessed via the short form of TEIQue, consisting of 30 statements responded to on a seven-point Likert scale (e.g., 'I often find it hard to understand other people'). Higher scores indicated a higher level of TEI. The test measured four factors (well-being, self-control, emotionality, sociability).

Beck Depression Inventory-II (BDI-II [37]; the Serbian version of the BDI II [38]. BDIII is a 21-item self-report instrument reflecting cognitive-affective as well as somatic aspects of depression. Higher scores suggested symptoms of depression.

Reactive-Proactive Aggression Questionnaire (RPAQ [39]). RPAQ is a 23-item measure, assessing aggression on a three-point Likert scale ranging from 0 (never) to 2 (always) (e.g., "How often have you used physical force to get others to do what you want"). In this study, we focused on the total aggression score. Higher scores indicated higher scores of aggression. The test items were adapted from English to Serbian using the double-translation procedure.

\subsection{Data analysis}

Primary analyses were performed using SPSS for Windows (version 26.0, SPSS Inc., Chicago, IL, USA). Descriptive statistics illustrated means and standard deviation scores on measured variables in the total sample, while correlations were tested using Pearson's correlation coefficient. Independent samples t-test was used to test group differences. Path Analysis (in AMOS, version 24) was conducted to test the theoretical model that predicts depressive or aggressive behavior from SPS with the emotional intelligence and decisionmaking styles as mediators. Specific indirect effects were estimated using the AMOS plugin [40]. Estimates of indirect effects were made using a bias-corrected bootstrap technique with 2000 samples and 95\% confidence intervals.

\section{Results}

Means, standard deviations, score ranges, as well as internal consistencies for all examined variables are shown in Table 1. Cronbach's alphas were mostly adequate and comparable to those obtained in previous research.

Table 1. Descriptive statistics and internal consistencies among variables.

\begin{tabular}{lccc}
\hline Variables & $\alpha$ & $\boldsymbol{M}(\mathrm{SD})$ & Min.-Max \\
\hline 1. SPS & 0.88 & $4.55(0.81)$ & $2.11-6.44$
\end{tabular}




\begin{tabular}{lccc} 
2.Depression & 0.90 & $9.62(8.08)$ & $0.00-52.00$ \\
3.Aggression & 0.86 & $10.62(6.15)$ & $0.00-46.00$ \\
4.Well-being & 0.81 & $5.41(1.01)$ & $2.50-7.00$ \\
5.Self-control & 0.73 & $4.62(1.06)$ & $1.33-7.00$ \\
6.Emotionality & 0.70 & $5.16(0.98)$ & $2.13-7.00$ \\
7.Sociability & 0.69 & $4.72(0.95)$ & $2.00-7.00$ \\
8.Spontaneous & 0.83 & $2.47(0.91)$ & $1.00-5.00$ \\
9.Rational & 0.79 & $4.08(0.66)$ & $1.80-5.00$ \\
10.Avoidant & 0.87 & $2.79(1.05)$ & $1.00-5.00$ \\
11.Intuitive & 0.86 & $3.69(0.82)$ & $1.17-5.00$ \\
12.Dependent & 0.81 & $3.61(0.80)$ & $1.40-5.00$ \\
13.Age & --- & $25.81(2.41)$ & $17-30$ \\
\hline
\end{tabular}

Statistically significant correlations were in expected directions: SPS was positively correlated with depression $(\mathrm{r}=.44, p<0.01)$, aggression $(\mathrm{r}=.21, p<0.01)$, avoidant $(\mathrm{r}=.26$, $p<0.01)$ and intuitive $(\mathrm{r}=.21, p<0.01)$ decision-making, while negatively with three out of four TEI factors: well-being $(\mathrm{r}=-.26, p<0.01)$, self-control $(\mathrm{r}=-.37, p<0.01)$ and sociability $(r=-.31, p<0.01)$. No correlation was registered between SPS and emotionality factor as well as between SPS and spontaneous decision-making.

Path analysis was utilized to test the theoretically assumed model which was corrected following obtained correlations. The path from SPS to depressive and aggressive types of reacting was outlined. Among the factors constituting TEI, as mediators were chosen: well-being, self-control, and sociability since they showed correlations with both SPS on the one hand and either depression or aggression on the other hand. Emotionality was not included as a mediator due to the lack of significant correlations. Avoidant decision-making style was added as a mediator for SPS and depression, and spontaneous decision-making as a mediator for SPS and aggression. Although, intuitive decision making showed correlations to SPS and aggression, and spontaneous decision-making did not correlate with SPS, the final model showed a better fit with the exclusion of intuitive and inclusion of spontaneous decision making style. Finally, such modified model (Figure 1) resulted in an acceptable fit: $\chi 2(5)=9.253$, ns; TLI $=.953$; $\mathrm{CFI}=.992$; $\mathrm{RMSEA}=.056$.

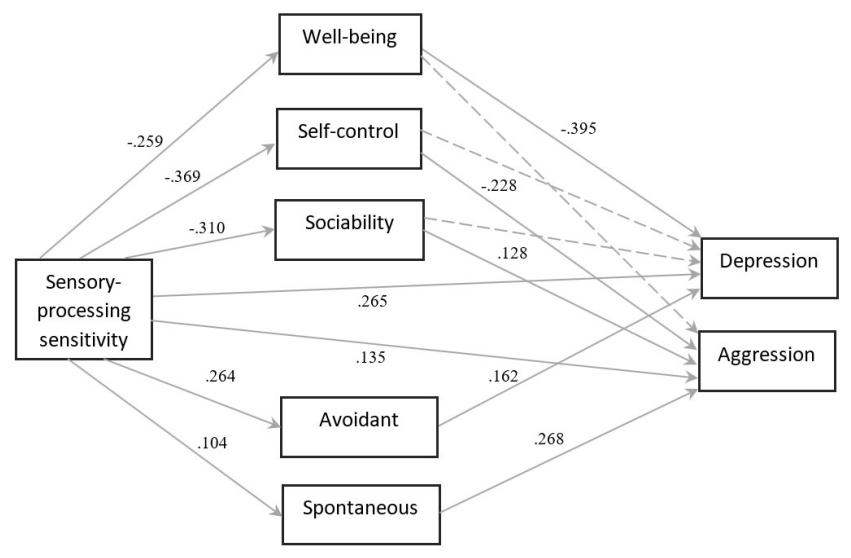

Figure 1. Results for the path analysis on total sample. Entries are standardized regression weights. Discontinuous lines represent non-significant paths. Non-Normed Fit Index TLI = .953; Comparative Fit Index CFI = .992; root mean square error of approximation RMSEA = .056; chisquare $\chi 2(5)=9.253, p>.05$.

SPS showed the following patterns of influence (Table 2): negative effects on wellbeing, self-control, and sociability; positive effects on avoidant and spontaneous decisionmaking; positive effects on depression and aggression. Among TEI factors: well-being negatively influenced depression but not aggression; self-control exerted a negative 
influence on aggression, but not on depression; sociability positively influenced aggression, but no effect was registered for depression. Out of decision-making styles, spontaneous decision-making positively affected aggression; while avoidant decision-making positively affected depression. Finally, partial mediators were revealed for depression: well-being $(\beta=.102, p<.001)$, and avoidant decision-making $(\beta=.043, p<.01)$. For aggression: self-control $(\beta=.084, p<.01)$.

Table 2. Paths, unstandardized regression weights, and standardized errors in total sample.

\begin{tabular}{|c|c|c|c|}
\hline \multicolumn{4}{|c|}{ Direct effects } \\
\hline Paths & $\mathrm{B}$ & S.E. & $95 \% \mathrm{CI}$ \\
\hline SPS $\rightarrow$ Well-being & $-.323^{* * *}$ & .07 & $-.472,-.190$ \\
\hline SPS $\rightarrow$ Self-control & $-.482^{* * *}$ & .07 & $-.625,-.337$ \\
\hline SPS $\rightarrow$ Sociability & $-.362^{* * *}$ & .07 & $-.503,-.233$ \\
\hline SPS $\rightarrow$ Avoidant & $.344^{* * *}$ & .08 & $.192, .495$ \\
\hline SPS $\rightarrow$ Depression & $2.645^{* * *}$ & .50 & $1.732,3.757$ \\
\hline SPS $\rightarrow$ Aggression & $1.026^{*}$ & .46 & $.-045,2.183$ \\
\hline Well-being $\rightarrow$ Depression & $-3.159^{* * *}$ & .42 & $-4.142,-2.251$ \\
\hline Self-control $\rightarrow$ Depression & -.672 & .43 & $-1.643, .211$ \\
\hline Self-control $\rightarrow$ Aggression & $-1.326^{* * *}$ & .40 & $-2.119,-.403$ \\
\hline \multicolumn{4}{|c|}{ Indirect effects } \\
\hline Paths & & $\mathrm{B}$ & $95 \%$ CI \\
\hline SPS $\rightarrow$ Well-being $\rightarrow$ De & ression & $1.022^{* * *}$ & $.615,1.606$ \\
\hline SPS $\rightarrow$ Avoidant $\rightarrow$ Dep & ession & $.427^{* *}$ & $.185, .765$ \\
\hline $\mathrm{SPS} \rightarrow$ Self-control $\rightarrow \mathrm{Ag}$ & ression & $.639^{* *}$ & $.279, .1 .057$ \\
\hline
\end{tabular}

Note. ${ }^{*} \mathrm{p}<.05 ;{ }^{* *} \mathrm{p}<.01 ;{ }^{* * *} \mathrm{p}<.001$

Regarding the gender differences, men registered higher scores in aggression (Mmen $=11.81$ and $\left.\mathrm{M}_{\text {women }}=9.87 ; \mathrm{t}(266)=2.54, p<.05\right)$ and self-control $\left(\mathrm{M}_{\text {men }}=4.93\right.$ and $\mathrm{M}_{\text {women }}=$ $4.42 ; \mathrm{t}(255)=4.00, p<.001)$, while women had higher scores in SPS ( $\mathrm{M}_{\text {women }}=4.73$ and $\mathrm{Mmen}_{\text {m }}$ $=4.26 ; \mathrm{t}(266)=-4.84, p<.001)$, depression $\left(\mathrm{M}_{\text {women }}=10.97\right.$ and $\mathrm{M}_{\text {men }}=7.48 ; \mathrm{t}(266)=-3.52$ $p<.001)$, emotionality $\left(\mathrm{M}_{\text {women }}=5.26\right.$ and $\left.\mathrm{M}_{\text {men }}=5.01 ; \mathrm{t}(266)=-1.98, p<.05\right)$ and intuitive decision-making $\left(\mathrm{M}_{\text {women }}=3.81\right.$ and $\left.\mathrm{M}_{\text {men }}=3.02 ; \mathrm{t}(266)=-3.13, p<.01\right)$.

Table 3. Correlations among study variables.

\begin{tabular}{lcccccccccccc}
\hline \multicolumn{1}{c}{ Variables } & $\mathbf{1}$ & $\mathbf{2}$ & $\mathbf{3}$ & $\mathbf{4}$ & $\mathbf{5}$ & $\mathbf{6}$ & $\mathbf{7}$ & $\mathbf{8}$ & $\mathbf{9}$ & $\mathbf{1 0}$ & $\mathbf{1 1}$ & $\mathbf{1 2}$ \\
\hline 1. SPS & --- & $.42^{* *}$ & .12 & $-.31^{* *}$ & $-.31^{* *}$ & .04 & $-.32^{* *}$ & .05 & .01 & $.25^{* *}$ & $.16^{*}$ & .13 \\
2.Depression & $.40^{* *}$ & --- & $.22^{* *}$ &.$- .61^{* *}$ & $-.39^{* *}$ & $-.22^{* *}$ & $-.43^{* *}$ & .13 & -.05 & $.36^{* *}$ & .02 & .14 \\
3.Aggression & $.45^{* *}$ & $.41^{* *}$ & --- & -.11 & $-.36^{* *}$ & $-.15^{* *}$ & -.01 & $.35^{* *}$ & -.11 & $.28^{* *}$ & $.29^{* *}$ & .11 \\
4.Well-being & -.19 & $-.40^{* *}$ & $-.20^{*}$ & --- & $.44^{* *}$ & $.26^{* *}$ & $.51^{* *}$ & .01 & .15 & $-.29^{* *}$ & $.16^{*}$ & -.10 \\
5.Self-control & $-.36^{* *}$ & $-.43^{* *}$ & $-.38^{* *}$ & $.50^{* *}$ & --- & $.20^{*}$ & $.52^{* *}$ & $-.31^{* *}$ & $.40^{* *}$ & $-.43^{* *}$ & .01 & $-.27^{* *}$ \\
6.Emotionality & -.05 & -.14 & $-.29^{* *}$ & .18 & $.29^{* *}$ & --- & $.36^{* *}$ & -.15 & .13 & $-.36^{* *}$ & $.18^{*}$ & .11 \\
7.Sociability & $-.28^{* *}$ & $-.34^{* *}$ & -.10 & $.36^{* *}$ & $.34^{* *}$ & $.45^{* *}$ & --- & -.08 & $.30^{* *}$ & $-.50^{* *}$ & .10 & -.14 \\
8.Spontaneous & $.22^{*}$ & $.26^{* *}$ & $.37^{* *}$ & -.10 & $-.42^{* *}$ & $-.21^{*}$ & -.06 & --- & $-.49^{* *}$ & $.22^{* *}$ & $.45^{* *}$ & -.12 \\
9.Rational & -.04 & -.18 & $-.21^{*}$ & $.33^{* *}$ & $.42^{* *}$ & $.29^{* *}$ & .11 & $-.53^{* *}$ & --- & $-.17^{*}$ & -.15 & .13 \\
10.Avoidant & $.28^{* *}$ & $.38^{* *}$ & .18 & -.15 & $-.38^{* *}$ & -.13 & $-.26^{* *}$ & $.26^{* *}$ & $-.26^{* *}$ & --- & .02 & $.28^{* *}$ \\
11.Intuitive & .18 & .16 & .070 & $.28^{* *}$ & -.15 & -.02 & .05 & $.58^{* *}$ & -.06 & .10 & -10 & .00 \\
12.Dependent & .06 & .02 & -.10 & $.21^{*}$ & -.17 & -.02 & -.12 & .09 & .04 & $.30^{* *}$ & .17 &.- .17 \\
\hline
\end{tabular}

Note. Men below diagonal and women above. ${ }^{*} \mathrm{p}<.05 ;{ }^{* *} \mathrm{p}<.01$.

Compared to the total sample, inspection of Pearson's correlations for men and women revealed several differences (Table 3). In the sample of men, SPS positively correlated with aggression (moderate) and spontaneous decision-making (small). On the other hand, in the sample of women SPS negatively correlated with well-being (moderate) and positively with intuitive decision-making (small). 
Testing for gender invariance showed the model as gender invariant $(\chi 2(13)=20.066$, $p>.05)$, meaning that the differences between men and women were not registered on the modal level. However, differences were registered on the path level (Figure 2). The positive relationship between SPS and spontaneous decision-making $(z=-1.23, p>.05)$, and SPS and aggression $(z=-2.91, p<.001)$ was only significant for men. Furthermore, the positive relationship between sociability and aggression was only significant for women $(z=0.9, p>$.05). Finally, the negative relationship between well-being and depression was stronger for women $(z=-2.12, p<.01)$.

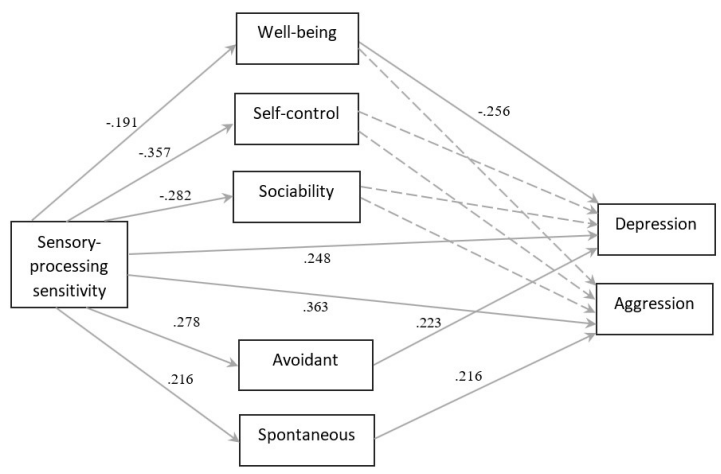

(a)

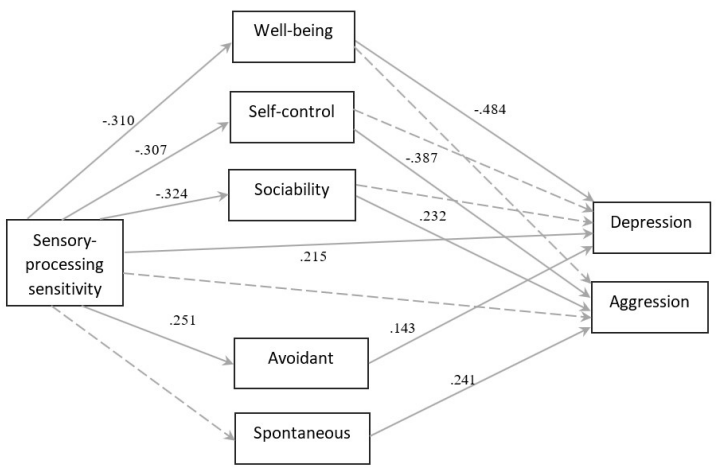

(b)

Figure 2. Results for the path analysis for: (a) men; (b) women. Entries are standardized regression weights. Discontinuous lines represent non-significant paths.

The estimates for both models are shown in Table 4. SPS was associated with and directly influenced problem behaviors, both depression (regardless of gender) and aggression (only for men). SPS indirectly influenced problem behaviors through mediating roles of TEI and decision-making styles. Specifically, partial mediation was registered for wellbeing and depression (regardless of gender), avoidant decision-making and depression (regardless of gender), spontaneous decision-making and aggression (only for men), and self-control and sociability and aggression (only for women).

Table 4. Paths, unstandardized regression weights, and standardized errors for men and women.

\begin{tabular}{|c|c|c|c|c|c|c|c|}
\hline \multicolumn{4}{|c|}{ Men } & \multicolumn{4}{|c|}{ Women } \\
\hline \multicolumn{8}{|c|}{ Direct effects } \\
\hline Paths & $\mathrm{B}$ & S.E. & $95 \% \mathrm{CI}$ & Paths & $\mathrm{B}$ & S.E. & $95 \% \mathrm{CI}$ \\
\hline SPS $\rightarrow$ Well-being & $-.211^{*}$ & .11 & $-.358,-.030$ & SPS $\rightarrow$ Well-being & $-.438^{* * *}$ & .11 & $-.607,-.270$ \\
\hline SPS $\rightarrow$ Self-control & $-.422^{* * *}$ & .11 & $-.643,-.218$ & SPS $\rightarrow$ Self-control & $-.432^{* * *}$ & .11 & $-.613,-.261$ \\
\hline SPS $\rightarrow$ Sociability & $-.334^{* *}$ & .11 & $-.502,-.156$ & SPS $\rightarrow$ Sociability & $-.399^{* * *}$ & .09 & $-.562,-.246$ \\
\hline SPS $\rightarrow$ Avoidant & $.354^{* *}$ & .12 & $.151, .555$ & SPS $\rightarrow$ Avoidant & $.353^{* * *}$ & .11 & $.162, .527$ \\
\hline SPS $\rightarrow$ Spontaneous & $.239^{* *}$ & .11 & $.026, .452$ & SPS $\rightarrow$ Spontaneous &. .064 & .10 & $-.090, .213$ \\
\hline SPS $\rightarrow$ Depression & $2.095^{* *}$ & .79 & $.984,3.301$ & SPS $\rightarrow$ Depression & $2.401^{* * *}$ & .70 & $1.304,3.574$ \\
\hline SPS $\rightarrow$ Aggression & $3.208^{* * *}$ & .79 & $1.420,5.225$ & SPS $\rightarrow$ Aggression & .436 & .53 & $-.389,1.245$ \\
\hline Well-being $\rightarrow$ Depression & $-1.951^{* *}$ & .71 & $-3.282,-.597$ & Well-being $\rightarrow$ Depression & $-3.813^{* * *}$ & .52 & $-4.800,-2.848$ \\
\hline Self-control $\rightarrow$ Depression & -.884 & .73 & $-2.025, .103$ & Self-control $\rightarrow$ Depression & -.390 & .55 & $-1.515, .609$ \\
\hline Self-control $\rightarrow$ Aggression & $-1.750^{* *}$ & .59 & $-2.960,-.376$ & Self-control $\rightarrow$ Aggression & $-1.955^{* * *}$ & .44 & $-2.789,-1.212$ \\
\hline Sociability $\rightarrow$ Aggression & $1.048^{*}$ & .48 & $.111,1.981$ & Sociability $\rightarrow$ Aggression & $1.337^{* *}$ & .48 & $.529,2.111$ \\
\hline Spontaneous $\rightarrow$ Aggression & $1.534^{* *}$ & .55 & $.464,2.690$ & Spontaneous $\rightarrow$ Aggression & $1.412^{* * *}$ & .43 & $.610,2.230$ \\
\hline
\end{tabular}

\begin{tabular}{|c|c|c|c|c|c|}
\hline Paths & $\mathrm{B}$ & $95 \%$ CI & Paths & $\mathrm{B}$ & $95 \% \mathrm{CI}$ \\
\hline SPS $\rightarrow$ Well-being $\rightarrow$ Depression & $.412^{*}$ & $.071,1.008$ & SPS $\rightarrow$ Well-being $\rightarrow$ Depression & $1.672^{* * *}$ & $.956,2.620$ \\
\hline SPS $\rightarrow$ Avoidant $\rightarrow$ Depression & $.524^{* *}$ & $.158,1.225$ & SPS $\rightarrow$ Avoidant $\rightarrow$ Depression & $.399^{*}$ & $.092, .976$ \\
\hline
\end{tabular}




\begin{tabular}{ll|l|lll} 
SPS $\rightarrow$ Spontaneous $\rightarrow$ Aggression & $.413^{*}$ & $.089,1.030$ & SPS $\rightarrow$ Self-comtrol $\rightarrow$ Aggression & $.844^{* * *}$ & $.455,1.441$ \\
& & SPS $\rightarrow$ Sociability $\rightarrow$ Aggression & $-.534^{* *}$ & $-.996,-.230$ \\
\hline Note. ${ }^{*} \mathrm{p}<.05 ;{ }^{* *} \mathrm{p}<.01 ;{ }^{* * *} \mathrm{p}<.001$ & &
\end{tabular}

\section{Discussion}

The present study aimed to explore the pathway model from sensory-processing sensitivity toward depression and aggression as the INT and EXT manifestations of distress. Mediating roles of TEI and general decision-making style, in addition to gender differences, were tested in different pathway models. The construed model excluded emotionality factor of TEI due to its lack of correlation with SPS. It is possible that emotionality includes some aspects at which high SPS individuals are assumed good at (e.g. empathy) and other aspects in which they may be subpar (e.g. relationship skills), thereby conceivably resulting in no correlation.

The suggested theoretical explanation for underlying mechanism behind SPS and depression was a secondary cognitive reactivity $[2,12]$. The authors argued that SPS was a sole factor responsible for amplification of negative sensory stimuli, resulting in secondary phenomenon of cognitive reactivity (maladaptive content and processes), which in turn leads to depression, anxiety and psychosomatic problems [12]. However, the provided model overvalues cognitive at the expense of emotive factors, and does not offer an explanation for the other type of problem behaviors - EXT behavior. Building on this model, we proposed that the negative stimuli amplification in both SPS core facets (cognitive and emotive processing), results in psychological distress, which may consequently be manifested in INT (depressive) or EXT (aggressive) behavior. In the following paragraphs we discussed the potential factors explored in this study from both cognitive (decision-making styles) and emotive (TEI) domain that may participate in psychological distress and problem behaviors.

Before moving on to the questions regarding the model, brief comments were provided for research variables and their correlations. Results from mean score differences and correlations mostly corresponded to previous research.

Firstly, in line with previous studies and as expected, men scored higher in aggression [9] and self-control [17], and women scored higher in SPS [30], depression [9], emotionality [18], while no difference was found in well-being [32]. Sociability showed no difference which differed from previous studies [17]. No differences in avoidant or spontaneous decision-making were registered which supported the studies that found no gender differences in decision-making styles [22], with the exception of intuitive decision-making, which was found to be greater in women in this study.

Secondly, expected pattern of associations was found in: SPS with depression [5]; and aggression [11]; well-being [18], self-control [18], sociability [18], but not in emotionality; avoidant and intuitive decision-making. Inspection of associations in the sample of men and women revealed important differences. SPS correlated with aggression and spontaneous decision-making only in men, while SPS showed association with well-being only in women. In light of the found gender differences, the interpretations were focused at the path level analysis.

\subsection{Sensitivity and depression: roles of TEI and decision-making style}

Sensitive individuals were more susceptible to depression, which is in line with previous studies $[5,6,10]$. The direct path from SPS toward depression still leaves the possibility of the inbuilt qualities of sensitive individuals to process stimuli more deeply. This result would support recent theories of depression as a secondary phenomenon of cognitive reactivity (i.e. maladaptive thought content and processes) to sensory information and related negative emotions [12]. However, the indirect path through well-being acted as an added factor, which was especially prominent in influencing depression in women, 
pointing to the relevance of emotional factor. Lower well-being (a less positive/optimistic representation of themselves, their past, present, and future) conforms with reports of depression [18], supporting Beck's notion that low self-regard is the core factor in depression [37]. Despite SPS being associated, in this and in previous studies, with poor stress management and difficulties in emotion regulation [16], as well as to social and communication deficits [6], self-control and sociability were not found to be mediators for depression in this sample.

Expectedly, avoidant decision-making acted as a partial mediator for SPS and depression, pointing to the possible differentiating effect on depression in contrast to an aggressive type of behavior. Being more sensitive seemed to have effects on cognitive style of avoidant decision-making which may have led to the mentioned problem behaviors, pointing to possible directionality of decision-making on depression, rather than the reverse [23]. This may reflect a greater sensitivity to risk present in depressed individuals $[25,26]$, which may have been emphasized in more sensitive individuals. It was also in line with the general avoidance strategy assumed present in more sensitive individuals [1]. These findings lend tentative support to our proposition of both emotional and cognitive factors participating in problem behaviors. Being more sensitive may amplify negative stimuli and overload emotive and cognitive capacities, slowing or inhibiting decisionmaking.

\subsection{Sensitivity and aggression: roles of TEI and decision-making}

A novel finding was reflected in SPS being associated with and influencing aggression, limited to men. This pathway raises questions about pause-to-check behaviors and general avoidance strategy assumed present in more sensitive individuals [1]. In contrast to women, SPS presented as a risk factor for aggression in the direct path in men. This result points to different strategies in dealing with psychological distress, with the tendency of men to choose EXT and the tendency of women to choose INT strategies [9,31].

Further differences in aggression in men and women were found when considering the indirect effects. A sense of immediacy and impulsiveness (reflected in spontaneous decision-making style) acted as a partial mediator in men, but not in women. These results were in line with the research supporting using different coping strategies when in distress [9]. In comparison to men, women were found to be more focused on mental states, both their own and others', and therefore engaged in aggression for reasons different than men [42]. Women seem to have a greater tendency to choose emotion-focusing while men seem to have a tendency to suppress or avoid dealing with emotions [9], which corresponds to greater INT problems found in women, and greater EXT problems found in men [31]. Greater emotion-focusing in women also corresponded to significantly greater effect of well-being factor in depression pathway in sensitive women found in this study. Conversely, men seem to engage in aggression impulsively and without considering mental states [42], which corresponded to EXT problems of suppressing and avoidance of emotion previously mentioned [9]. Avoidant and spontaneous decision-making styles, supported the idea of impaired decision-making amplifying the effects toward INT or EXT problems [24].

In partial accordance to previous studies [20], lower emotion regulation and impulse control (reflected in lower self-control) and greater social competence and affecting the emotions of others (reflected in higher sociability) was predictive of aggression, although only in women. Lower self-control has resemblance to impulsivity which has been previously connected to aggression [28]. Greater sociability may be suggested to have been utilized for attaining and maintaining social status [41]. An interesting relationship emerged with SPS negatively affecting self-control and sociability [16], and while selfcontrol negatively affected aggression, sociability positively affected aggression in women. Being a more sensitive woman seems to lower sociability thereby possibly acting as a suppressive factor for aggression in women. These results seemed to point to different 
TEI profiles with respect to gender [21]. However, the interpretation of this finding was restricted by both the lack of research in this area [21] and the sample size of the current study. A greater sample may point to the importance of such factors in men as well.

\subsection{Limitations and future directions.}

Firstly, this study was conducted on a community sample, limiting generalizability and suggesting the need for a clinical sample with respect to psychological distress and problem behaviors. Further restrictions were posed by a relatively small sample of men. Moreover, significant effects with specified directions obtained using path analysis for testing do not exclude the possibility of other good-fitting alternative models with differently proposed links and directions and thus imposing limits to causal interpretations. Longitudinal research would be adequate for this aim. Construal of the model in this study was guided by the suggested theoretical model of SPS and psychological distress [12] with the choice of cognitive and emotive variables as core facets of SPS, that were pertinent to problem behaviors.

An impetus for further research avenues may thus be twofold. Considering the supportive evidence gained from this pilot study, further cognitive and emotive factors warrant exploration in the relation of SPS and problem behaviors. Replication of these findings on a broader sample is also advised. Additionally, such models are adequate at explaining the bottom-up processing (from the environmental stimuli to cognitive and emotional factors), but may be limited at explaining the top-down processing (from cognitive and emotional factors toward stimuli) [21]. Not all sensitive individuals end up in a disadvantageous mental state [1-3]. A question of supportive and adverse environments should be reintroduced and expanded beyond early parental [43], and toward cultural contexts, with the inclusion of gender differences. The potentially relevant variable may also be found in a personality structure of the individual.

\section{Conclusions}

Despite limitations, this study was the first to extend SPS research to externalizing type of reacting through aggression. Moreover, representing the core facets of SPS, the study tested both emotional (TEI) and cognitive (decision-making styles) mediators, considered relevant and participating in problem behavior. Gender differences were also highlighted. In essence, this study suggested that higher sensitivity coupled with distress may manifest in both depression (INT) and aggression (EXT). With respect to depression, both emotive factor of well-being and a cognitive factor of avoidant decision-making participated as partial mediators for depression, regardless of gender. Self-control and sociability did not participate as mediators for depression. Concerning aggression, only selfcontrol participated as a partial mediator. Additionally, gender differences shed more light in the pathways of aggression. SPS was directly associated with aggression only in men. Indirect paths also differed, with spontaneous decision-making (characterized by impulsiveness and urgency) showing partial mediation only for men, while self-control and sociability showing partial mediation only for women. This finding corroborates different reasons behind aggression in men and women. These results were in line with the theoretical proposition that SPS acts as an amplifier of negative stimuli, resulting in diminishing of the both emotional and cognitive capacities of the individual, possibly responsible for problem behaviors, both depression and aggression.

Supplementary Materials: No supplementary materials.

Author Contributions: N.D. and S.P. contributed equally to this paper and can be considered cofirst authors. Conceptualization, formal analysis, writing-original draft preparation N.D., S.P.; methodology, writing - review and editing, N.D., S.P. and J.M.M.; investigation, N.D., S.P.; data curation, N.D., S.P. All authors have read and agreed to the published version of the manuscript. 
Funding: This study was conducted as a part of the project of the Institute of criminological and sociological research, Belgrade, Serbia, funded by Ministry of Education, Science and Technological Development of Republic of Serbia.

Institutional Review Board Statement: The study was conducted according to the guidelines of the Declaration of Helsinki and approved by the Research and Institutional Review Board of the Faculty of Philosophy, University of Belgrade, Serbia on 30/07/2020.

Informed Consent Statement: Informed consent was obtained for all subjects involved in the study.

Data Availability Statement: Database is available on request by the first author.

Acknowledgments: No acknowledgements.

Conflicts of Interest: The authors declare no conflict of interest.

\section{References}

1. Aron, Elaine N., and Arthur Aron. Sensory-processing sensitivity and its relation to introversion and emotionality. Journal of personality and social psychology. 1997, 73, 345. https://doi.org/10.1037/0022-3514.73.2.345

2. Greven, Corina U., Francesca Lionetti, Charlotte Booth, Elaine N. Aron, Elaine Fox, Haline E. Schendan, Michael Pluess et al. Sensory processing sensitivity in the context of environmental sensitivity: A critical review and development of research agenda. Neuroscience \& Biobehavioral Reviews 2019, 98, 287-305. https://doi.org/10.1016/j.neubiorev.2019.01.009

3. Aron, Elaine N., Arthur Aron, and Jadzia Jagiellowicz. Sensory processing sensitivity: A review in the light of the evolution of biological responsivity. Personality and Social Psychology Review. 2012, 163, 262-282. https://doi.org/10.1177/1088868311434213

4. Boterberg S, Warreyn P. Making sense of it all: The impact of sensory processing sensitivity on daily functioning of children. Personality and Individual Differences. 2016, 92, 80-6. https://doi.org/10.1016/j.paid.2015.12.022

5. Liss M, Timmel L, Baxley K, Killingsworth P. Sensory processing sensitivity and its relation to parental bonding, anxiety, and depression. Personality and individual differences. 2005, 39, 1429-39. https://doi.org/10.1016/j.paid.2005.05.0077

6. Liss M, Mailloux J, Erchull MJ. The relationships between sensory processing sensitivity, alexithymia, autism, depression, and anxiety. Personality and individual differences. 2008, 45, 255-9. https://doi.org/10.1016/i.paid.2008.04.009

7. Neal JA, Edelmann RJ, Glachan M. Behavioural inhibition and symptoms of anxiety and depression: Is there a specific relationship with social phobia?. British Journal of Clinical Psychology. 2002, 41, 361-74. https://doi.org/10.1348/014466502760387489

8. Benham G. The highly sensitive person: Stress and physical symptom reports. Personality and individual differences. 2006, 40, 1433-40. https://doi.org/10.1016/j.paid.2005.11.021

9. Nolen-Hoeksema S. Emotion regulation and psychopathology: The role of gender. Annual review of clinical psychology. 2012, 8 , 161-87. https://doi.org/10.1146/annurev-clinpsy-032511-143109

10. Yano K, Oishi K. The relationships among daily exercise, sensory-processing sensitivity, and depressive tendency in Japanese university students. Personality and Individual Differences. 2018, 127, 49-53. https://doi.org/10.1016/j.paid.2018.01.047

11. Slagt M, Dubas JS, van Aken MA, Ellis BJ, Deković M. Sensory processing sensitivity as a marker of differential susceptibility to parenting. Developmental psychology. 2018, 54, 543. https://doi.org/10.1016/j.jecp.2016.10.004

12. Wyller HB, Wyller VB, Crane C, Gjelsvik B. The relationship between sensory processing sensitivity and psychological distress: A model of underpinning mechanisms and an analysis of therapeutic possibilities. Scandinavian Psychologist. 2017, 23, 4.

13. Petrides KV, Mikolajczak M, Mavroveli S, Sanchez-Ruiz MJ, Furnham A, Pérez-González JC. Developments in trait emotional intelligence research. Emotion review. 2016, 8, 335-41. https://doi.org/10.1177/1754073916650493

14. Petrides KV. Psychometric properties of the trait emotional intelligence questionnaire (TEIQue). In Assessing emotional intelligence 2009 (pp. 85-101). Springer, Boston, MA.

15. Sobocko K, Zelenski JM. Trait sensory-processing sensitivity and subjective well-being: Distinctive associations for different aspects of sensitivity. Personality and individual differences. 2015, 83, 44-9. https://doi.org/10.1016/j.paid.2015.03.045

16. Brindle K, Moulding R, Bakker K, Nedeljkovic M. Is the relationship between sensory-processing sensitivity and negative affect mediated by emotional regulation?. Australian Journal of Psychology. 2015, 67(4), 214-21. https://doi.org/10.1111/ajpy.12084

17. Gugliandolo MC, Costa S, Cuzzocrea F, Larcan R, Petrides KV. Trait emotional intelligence and behavioral problems among adolescents: A cross-informant design. Personality and Individual Differences. 2015, 74, 16-21. https://doi.org/10.1016/j.paid.2014.09.032 
18. Mikolajczak M, Luminet O, Leroy C, Roy E. Psychometric properties of the Trait Emotional Intelligence Questionnaire: Factor structure, reliability, construct, and incremental validity in a French-speaking population. Journal of personality assessment. 2007, 88, 338-53. https://doi.org/10.1080/00223890701333431

19. Mavroveli S, Petrides KV, Rieffe C, Bakker F. Trait emotional intelligence, psychological well-being and peer-rated social competence in adolescence. British journal of developmental psychology. 2007, 25, 263-75. https://doi.org/10.1348/026151006X118577

20. Milojević S, Dimitrijević AA, Marjanović ZJ, Dimitrijević A. Bad past, gloomy future: The trait emotional intelligence profile of juvenile offenders. Personality and Individual Differences. 201, 94, 295-8. https://doi.org/10.1016/j.paid.2016.01.040

21. Bacon AM, Lenton-Maughan L, May J. Trait emotional intelligence and social deviance in males and females. Personality and Individual Differences. 2018, 122, 79-86. https://doi.org/10.1016/j.paid.2017.10.015

22. Scott SG, Bruce RA. Decision-making style: The development and assessment of a new measure. Educational and psychological measurement. 1995, 55, 818-31. https://doi.org/10.1177/0013164495055005017

23. Leykin Y, DeRubeis RJ. Decision-making styles and depressive symptomatology: Development of the Decision Styles Questionnaire. Judgment and Decision making. 2010, 5, 506.

24. Flouri E, Ruddy A, Midouhas E. Maternal depression and trajectories of child internalizing and externalizing problems: the roles of child decision making and working memory. Psychological medicine. 2017, 47, 1138-48. https://doi.org/10.1017/S0033291716003226

25. van Randenborgh A, de Jong-Meyer R, Hüffmeier J. Decision making in depression: differences in decisional conflict between healthy and depressed individuals. Clinical psychology \& psychotherapy. 2010, 17, 285-98.

26. Smoski MJ, Lynch TR, Rosenthal MZ, Cheavens JS, Chapman AL, Krishnan RR. Decision-making and risk aversion among depressive adults. Journal of behavior therapy and experimental psychiatry. 2008, 39, 567-76. https://doi.org/10.1016/j.jbtep.2008.01.004

27. Kuin N, Masthoff E, Kramer M, Scherder E. The role of risky decision-making in aggression: A systematic review. Aggression and violent behavior. 2015, 25, 159-72. https://doi.org/10.1016/j.avb.2015.07.018

28. Franken IH, van Strien JW, Nijs I, Muris P. Impulsivity is associated with behavioral decision-making deficits. Psychiatry research. 2008, 158, 155-63. https://doi.org/10.1016/j.psychres.2007.06.002

29. Endres MJ, Donkin C, Finn PR. An information processing/associative learning account of behavioral disinhibition in externalizing psychopathology. Experimental and clinical psychopharmacology. 2014, 2, 122. https://doi.org/10.1037/a0035166

30. Kibe C, Suzuki M, Hirano M, Boniwell I. Sensory processing sensitivity and culturally modified resilience education: Differential susceptibility in Japanese adolescents. PloS one. 2020, 15, e0239002. https://doi.org/10.1016/j.avb.2015.07.018

31. Piqueras JA, Mateu-Martínez O, Cejudo J, Pérez-González JC. Pathways into psychosocial adjustment in children: modeling the effects of trait emotional intelligence, social-emotional problems, and gender. Frontiers in psychology. 2019, $12,507$. https://doi.org/10.3389/fpsyg.2019.00507

32. Martskvishvili K, Arutinov L, Mestvirishvili M. A psychometric investigation of the Georgian version of the Trait Emotional Intelligence Questionnaire. European Journal of Psychological Assessment. 2013. https://doi.org/10.1027/1015-5759/a000135

33. Gomez-Baya D, Mendoza R, Paino S, de Matos MG. Perceived emotional intelligence as a predictor of depressive symptoms during mid-adolescence: A two-year longitudinal study on gender differences. Personality and individual differences. 2017, 104, 303-12. https://doi.org/10.1016/j.paid.2016.08.022

34. Curşeu PL, Schruijer SG. Decision styles and rationality: An analysis of the predictive validity of the General Decision-Making Style Inventory. Educational and Psychological Measurement. 2012, 72, 1053-62. https://doi.org/10.1177/0013164412448066

35. Johnson JE, Powell PL. Decision making, risk and gender: Are managers different?. British journal of management. 1994, 2, 12338. https://doi.org/10.1111/j.1467-8551.1994.tb00073.x

36. Jolić-Marjanović Z, Altaras-Dimitrijević A. Reliability, construct and criterion-related validity of the Serbian adaptation of the Trait Emotional Intelligence Questionnaire (TEIQue). Psihologija. 2014, 47, 249-62. https://doi.org/10.2298/PSI1402249J

37. Beck AT, Steer RA, Ball R, Ranieri WF. Comparison of Beck Depression Inventories-IA and-II in psychiatric outpatients. Journal of personality assessment. 1996, 67(3), 588-97. https://doi.org/10.1207/s15327752jpa6703_13

38. Novović Z, Mihić L, Tovilović S, Jovanović V, Biro M. Psychometric characteristics of the Beck depression inventory on a Serbian student sample. Psihologija. 2011, 44, 225-43. https://doi.org/10.2298/PSI1103225N

39. Raine A, Dodge K, Loeber R, Gatzke-Kopp L, Lynam D, Reynolds C, Stouthamer-Loeber M, Liu J. The reactive-proactive aggression questionnaire: Differential correlates of reactive and proactive aggression in adolescent boys. Aggressive Behavior: Official Journal of the International Society for Research on Aggression. 2006, 32, 159-71. https://doi.org/10.1002/ab.20115

40. Gaskin J, Lim J. Multigroup analysis, amos plugin. Gaskination's StatWiki. 2018.

41. Cohn A, Zeichner A. Effects of masculine identity and gender role stress on aggression in men. Psychology of Men E Masculinity. 2006, 4, 179. https://doi.org/10.1037/1524-9220.7.4.179

42. Protic S, Wittmann L, Taubner S, Dimitrijevic A. Differences in attachment dimensions and reflective functioning between traumatized juvenile offenders and maltreated non-delinquent adolescents from care services. Child abuse $\mathcal{E}$ neglect. 2020, 103, 104420. https://doi.org/10.1016/j.chiabu.2020.104420

43. Aron EN, Aron A, Davies KM. Adult shyness: The interaction of temperamental sensitivity and an adverse childhood environment. Personality and Social Psychology Bulletin. 2005, 31, 181-97. https://doi.org/10.1177/0146167204271419 